\title{
Chapter 18 \\ Texture Transition in Steel ST37K, in situ Measurement at High Temperatures Using High-Energy X-rays
}

\author{
H.-G. Brokmeier, S.B. Yi, and J. Homeyer
}

\begin{abstract}
High energy X-rays are well known due to there high penetration power particular in materials testing devices. For diffraction experiments high energy X-rays with more than $50 \mathrm{keV}$ can be obtained at storage rings or using a tungsten X-ray tube. According to the high penetration power, these beamlines offer a very high photon flux and an excellent brilliance. That means measurements can be carried out fast. As an example, the complete texture measurement at one position of a steel shaft with $34 \mathrm{~mm}$ in diameter has taken 45 minutes nondestructively. On the other hand the high photon flux allows to measure foils or thin wires down to $50-100 \mu \mathrm{m}$. These new and fast options make it possible to measure in situ textures under tension, compression and at high temperatures. We have used $100 \mathrm{keV} \mathrm{X}$-ray to measure the texture transition as well as the phase transition in a steel sample. The experiments were done at the high energy beamline BW5 (Hasylab at Desy/Hamburg). $100 \mathrm{keV}$ X-rays have a wavelength of $0.1240 \AA$ which means due to the Bragg's law very low scattering angles. Using a MAR345 image plate detector one obtains a set of complete Debye-Scherrer cones in a $2 \theta$-range of $7^{\circ}$ in about $1 \mathrm{sec}$. At room temperature we found $100 \%$ ferrite. During heating up till the austenite region we were able to investigate the thermal expansion and the texture relation between ferrite and austenite, which follows in our case the Kurdjumov-Sachs model. Furthermore, the program pack-
\end{abstract}

H.-G. Brokmeier

Institute of Materials Science and Engineering TU Clausthal, Agricolastr. 6, D-38678 Clausthal-Zellerfeld, and

GKSS Forschungszentrum, Max-Planck-Str. 1, D-21502 Geesthacht, Germany

S.B. Yi

Institute of Materials Science and Engineering TU Clausthal, Agricolastr. 6, D-38678 Clausthal-Zellerfeld

J. Homeyer

Hasylab at Desy, Notkestraße 85, 22607 Hamburg, Germany

A. Haldar, S. Suwas, and D. Bhattacharjee (eds.), Microstructure and Texture in Steels, 
age MAUD offers the possibility to follow the phase transition, so that the composition at all temperatures can be documented during heating. It has to be pointed out that the texture influence on the quantitative phase analysis can be included by MAUD, so that even for strong crystallographic textures the relation ferrite/austenite can be given very well.

\subsection{Synchrotron Radiation}

In materials science high energy X-rays are long-time restricted to material testing. Standard material testing devices operate from $120 \mathrm{keV}$ to $450 \mathrm{keV}$ due to their application field for non-destructive testing (NDT), such as failure analysis in pipelines, quality control in wheel rims and many other technical products. Typical diffraction experiments for phase, texture and strain analysis were carried out by conventional $\mathrm{X}$-ray equipments using $\mathrm{Cu}$-, $\mathrm{Co}$ or $\mathrm{Cr}$-tubes with much lower $\mathrm{X}$-ray energies. Firstly, the development of synchrotron storage rings opens the field of diffraction for high energy X-rays, which ranges from about $50 \mathrm{keV}$ up to $450 \mathrm{keV}[1,2,3]$.

One main advantage of high energy $\mathrm{X}$-rays is the penetration power. Figure 18.1 shows the penetration length into some metals $(\mathrm{Mg}, \mathrm{Al}, \mathrm{Ti}$ and $\mathrm{Fe})$ in the range up to $250 \mathrm{keV}$. The penetration length is given in $\mathrm{cm}$ for a loss of $50 \%$ of the primary beam intensity. The two marked lines present on one hand $\mathrm{Cu} \mathrm{Ka} \mathrm{X}$-rays with $9 \mathrm{keV}$ as $\mathrm{X}$-ray tube voltage $(1.5418 \AA)$ and on the other hand our mainly used synchrotron wavelength of $0.124 \AA$ wavelength with $100 \mathrm{keV}$. In the case of $200 \mathrm{keV} \mathrm{X}$-rays the penetration length is in the same order as for thermal neutrons, which are well known in materials science applications (texture and strain analyses) as high penetrating radiation. $[4,5]$.

In comparison with laboratory scale X-ray devices a storage ring has a much higher photon flux. But to get an optimized flux for the so called hard X-rays one

Fig. 18.1 X-ray transmission of $\mathrm{Fe}, \mathrm{Ti}, \mathrm{Al}$ and $\mathrm{Mg}$ as function of the energy.

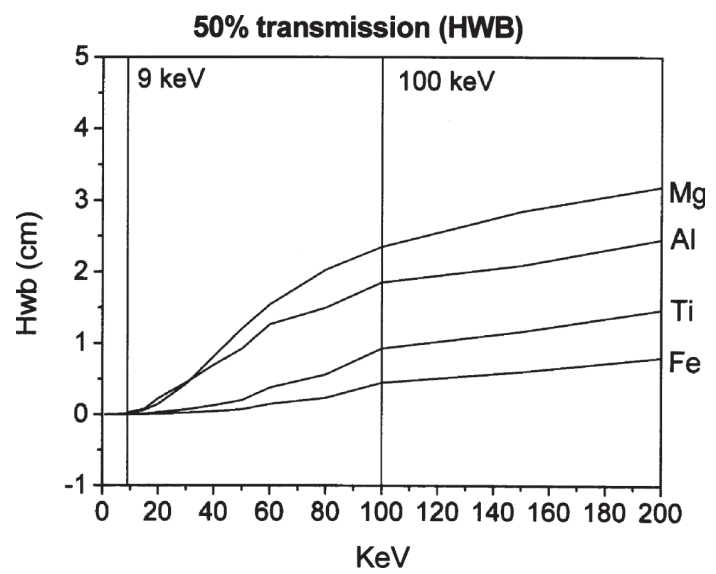


need special arrangements of the wiggler, which is realized recently at the new hard wiggler beamline HARWI-II of the GKSS Research Center Geesthacht $\mathrm{GmbH}$ at the Hasylab storage ring Doris, DESY-Hamburg (Germany). With BW5 and Harwi-II two high energy X-ray beamlines are available at Hasylab/DESY Hamburg.

According to the much higher flux of these synchrotron beamlines one gets a fantastic brilliance. High brilliance means an excellent parallel photon beam, so that only those grains are in reflections condition, which have the ideal orientation to the incoming beam. Even small misorientations of crystallites to the Bragg condition results in the fact that these crystallites cannot be seen, like single crystal orientation determination.

The combination of a high penetration power, a high photon flux and an excellent brilliance offers new options for diffraction experiments in materials science applications. Firstly, a high photon flux results in a good counting statistics and short counting times [3]. Short counting times are the bases of time resolved investigations (recrystallization or phase transitions) and for in situ studies $[6,7,8]$. Secondly, the excellent brilliance combined with the high photon flux allows local measurements in the $\mu \mathrm{m}$-scale [8]. Thirdly, the high penetration power on one hand and the high photon flux on the other hand made it possible to measure rather small samples such as wires or foils [9] but also relatively large samples of some $\mathrm{cm}$ in diameter such as a steel shaft. In Fig. 18.2 special sample holders including

Fig. 18.2 Two examples of for successful synchrotron texture analysis, (a) Fe-foil of $10 \mu \mathrm{m}$ thickness and (c) steel shaft of $34 \mathrm{~mm}$ thickness including two (110) pole figures (b) and (d).

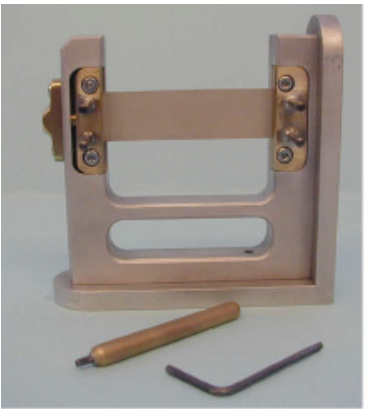

(a)

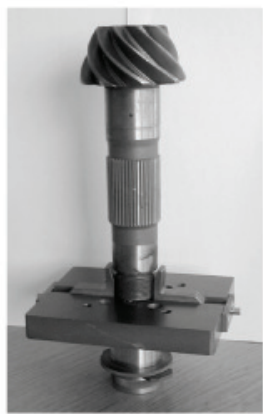

(c)

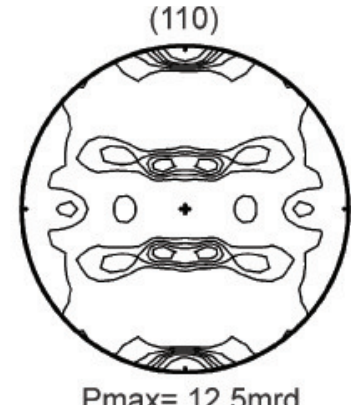

(b)

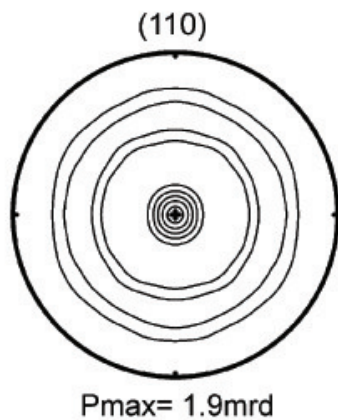

(d) 
a thin Fe-foil of $10 \mu \mathrm{m}$ (Fig. 18.2 (a)) and a comparably heavy steel sample of $34 \mathrm{~mm}$ thickness and $100 \mathrm{~mm}$ high (Fig. 18.2 (c)) are shown. Both samples were measured at the high energy beamline BW5 and evaluated by the iterative series expansion method for quantitative texture analysis. In both cases a beam cross section of $1 \times 1 \mathrm{~mm}^{2}$ was used. The recalculated (110) pole figures are presented in Fig. 18.2 (b) (Fe-foil) and in Fig. 18.2 (d) (steel shaft).

\subsubsection{Hard X-ray Instrumentation}

The beam path of a high energy synchrotron diffractometer is close to a conventional pin whole device, which is long-time used by film techniques [10]. A system of magnets called bending magnet, undulator or wiggler generates a synchrotron beam over a white range of energies between $1 \mathrm{keV}$ up to $400 \mathrm{keV}$. Firstly a filter and secondly a monochromator are used to produce a high intense monochromatic beam. Figure 18.3 (a) shows the beam path at BW5 including the monochromator tank (yellow) and the sample stage with an Eulerian cradle. The heavy sample stage is able to carry different equipments such as furnaces, loading cells and so on. Following the pin-whole camera technique the monochromatic beam is guided by different diaphragms (D), which can be driven automatically to get a very precise beam cross section. The pin-whole technique in conventional X-ray diffractometry allows the transmission as well as the reflection method. According to the high penetration power of high-energy X-rays, the transmission method is preferred, as shown in Fig. 18.3 (b).

Due to the high energy of about $100 \mathrm{keV}$ the wavelength with $0.124 \AA$ is short and consequently one can get a set of complete Debye-Scherrer cones on an area detector. In Fig. 18.4 (a) one can see the image plate picture of Fe-powder obtained in $5 \mathrm{sec}$. using a MAR345 image plate detector. All Debye-Scherrer rings show random orientate grains with nearly identical intensities around the rings.

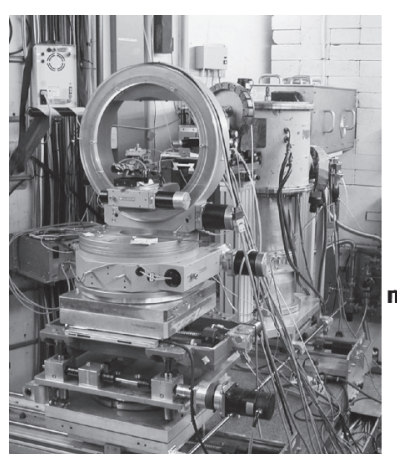

(a)

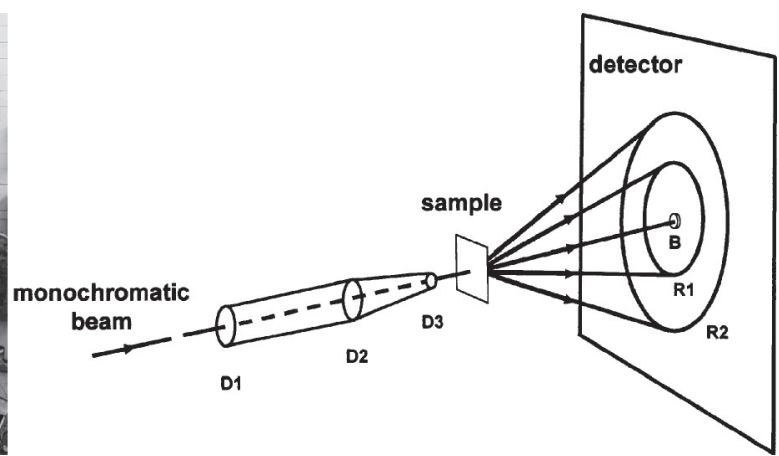

(b)

Fig. 18.3 Beam path of a synchrotron diffractometer (a) BW5-instrument; (b) pin whole technique (D - diaphragms, $\mathrm{R}$ - Bragg-reflections on the detector, B - beam stop). 


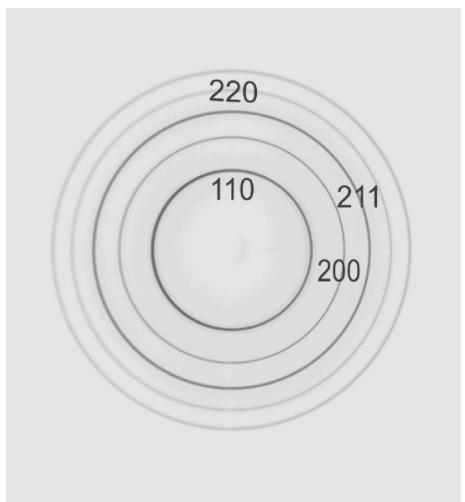

(a)

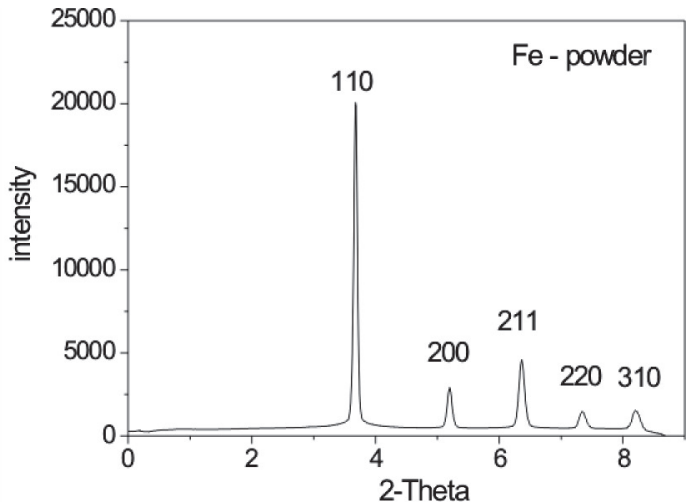

(b)

Fig. 18.4 Diffraction pattern of Fe-powder; (a) complete Debye-Scherrer rings on an area detector, (b) sum pattern after integration over the complete Debye-Scherrer rings.

The sum-diffraction pattern calculated by integration along the rings is given in Fig. 18.4 (b) with (110) as dominating reflection.

\subsubsection{Texture Measurements by High Energy Synchrotron Radiation}

The working horse for texture measurements is still based on laboratory X-rays, which is described in many papers [11]. The principles of the pole figure measurement using synchrotron radiation is very close to that of conventional X-rays with an X-ray tube. That means, the texture analysis using high energy X-rays with a two-dimensional position sensitive detector is firstly a step back to the early methods well known as film techniques $[12,13,14]$ and secondly a big step forward to combine a brilliant radiation source with modern detectors such as CCDcameras and image-plate detectors. Experiments recently carried out at Harwi-II (Hasylab@Desy) using a new MAR555 detector, take only 5-10 min for a complete texture measurement.

Two main differences exist between X-ray energies of $100 \mathrm{keV}(\lambda=0.124 \AA)$ and of $9 \mathrm{keV}(\lambda=1.5418 \AA)$. In both cases a set of complete Debye-Scherrer rings can be detected simultaneously by an area detector, depending on the sample to detector distance (see Fig. 18.4 (a)). The stereographic projection of one DebyeScherrer cone in the case of perpendicular transmission is shown in Fig. 18.5 (a). The circle in the stereographic projection is connected with the reflection angle $2 \theta$ and the radius of the projected circle in the 'pole figure' is $90^{\circ}-\theta$.

On one hand one can conclude that for very low $\theta$-values only an $\omega$-rotation is necessary (Fig. 18.5 (b) and Fig. 18.5 (c)) but that on the other hand a blind area for higher $\theta$-values exists (13). Moreover, due to the high penetration power of 
synchrotron radiation one obtains complete pole figures in the case of round samples. The second advantage of $100 \mathrm{keV}$ synchrotron radiation is also related to the low scattering angle because a low scattering angle needs only a comparable small opening angle of shielding and so on. In comparison with only one rotation axis this allows the construction of special loading cells and furnaces which are not available for conventional $\mathrm{X}$-rays.

The high brilliance of high energy synchrotron radiation yields to a very special pole figure window [15]. Due to the quantitative texture itself $\Delta \omega$ has to be cho-

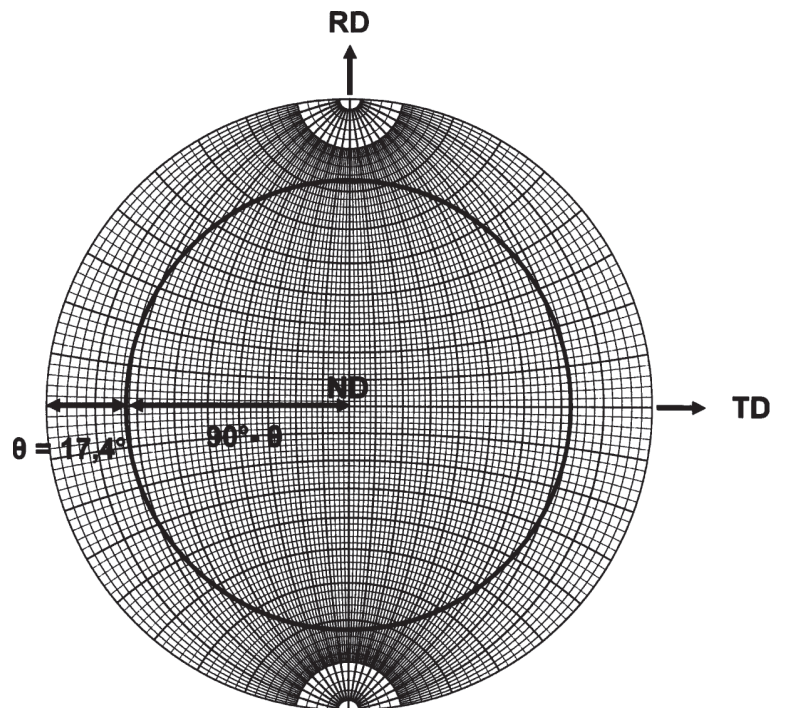

(a)

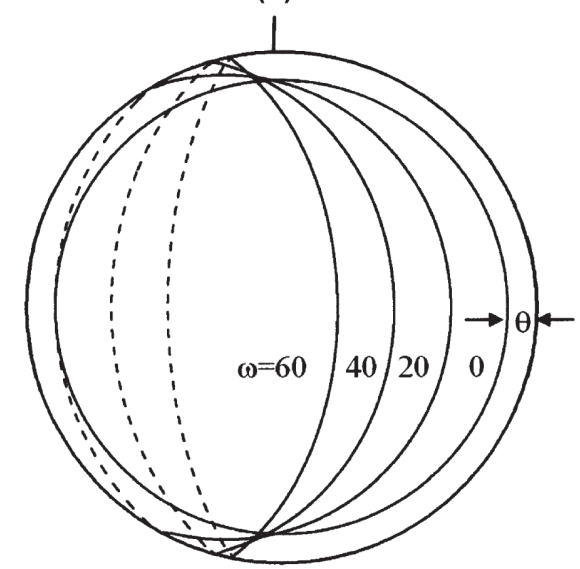

(c)

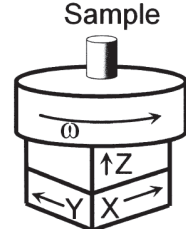

(b)

Fig. 18.5 (a) Pole figure with the projection of one Debye-Scherrer ring with $\theta=17.4^{\circ}$ (b) sample table with only one $\omega$-rotation table (c) projection of the Debye-Scherrer ring for different $\omega$-rotations. 
sen, so that the texture sharpness has to be reproduced correctly. The nearly parallel synchrotron beam allows the investigation of very sharp textures if $\omega$ is scanned in small steps $\left(\Delta \omega=1^{\circ}\right.$ or less). Another point of interest is that for low symmetric textures $\omega$ must be scanned from $-90^{\circ}$ till $+90^{\circ}$. A typical beam size is between $1 \mathrm{~mm} \times 1 \mathrm{~mm}$ and $1 \mathrm{~mm} \times 4 \mathrm{~mm}$, which is comparably small to neutron diffraction the favoured method for global texture measurements. In the case of round samples, see Fig. 18.2 (c), one need in single phase materials no materials dependent corrections. For other sample shapes (rectangular sheets, semi finished products) corrections for absorption and constant volume has to be carried out.

As already pointed out synchrotron radiation is much more intense than conventional X-rays and therefore the total counting can be much lower. Thus time resolved in situ texture measurements are possible, such as in situ investigations of texture evolutions under tensile load [16], compressive load [17] or high temperatures [18].

\subsection{High Temperature Measurements by High-Energy Synchrotron Radiation}

Due to the high photon intensity of synchrotron radiation fast in situ measurements can be carried restricted mainly by the read-out time of the detector and the heating rate of the furnace. Moreover, the high penetration power allows the transmission of furnace wall and heating shield without any problems.

\subsubsection{Sample Description and Experimental Conditions}

The investigated test sample was a steel ST37 K with a chemical composition of $0.10 \mathrm{C}, 0.01 \mathrm{Si}, 0.72 \mathrm{Mn}, 0.027 \mathrm{P}, 0.027 \mathrm{~S}$ balanced Fe. For the synchrotron measurement a stick of $5 \times 5 \mathrm{~mm}^{2}$ with a high of $10 \mathrm{~mm}$ was prepared. In addition neutron diffraction was performed to control the results of room temperature measurements. Therefore, a sample cube of $10 \times 10 \times 10 \mathrm{~mm}^{3}$ was prepared. A dome furnace (Fig. 18.6 ), constructed by Hasylab, was positioned on the omega table, see Fig. 18.5 (b).

The couple of the dome furnace was $\mathrm{SiO}_{2}$-glas, so that for all sample rotations in omega and a wide range of sample tilt identical conditions exist. Heating was realized by a graphite foil heating system (Fig. 18.6 (b)). Graphite as a light element has very high transmission for high-energy X-rays so that the graphite was not seen by the synchrotron radiation. The whole area under the graphite foil has nearly identical temperature. In the centre of the graphite foil the sample sheet was positioned. To control the temperature a thermocouple was fixed at the lower part of the sample, which is not in the beam. In Fig. 18.6 (c) one can see the furnace as central part of the instrumentation with the MAR345 area detector in the back. 


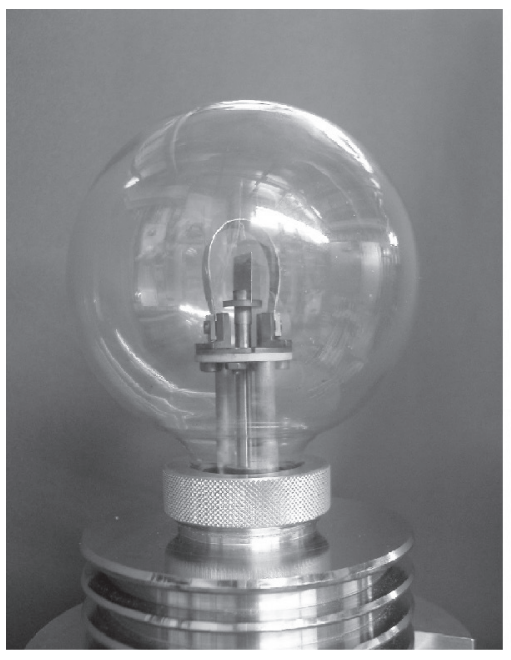

(a)

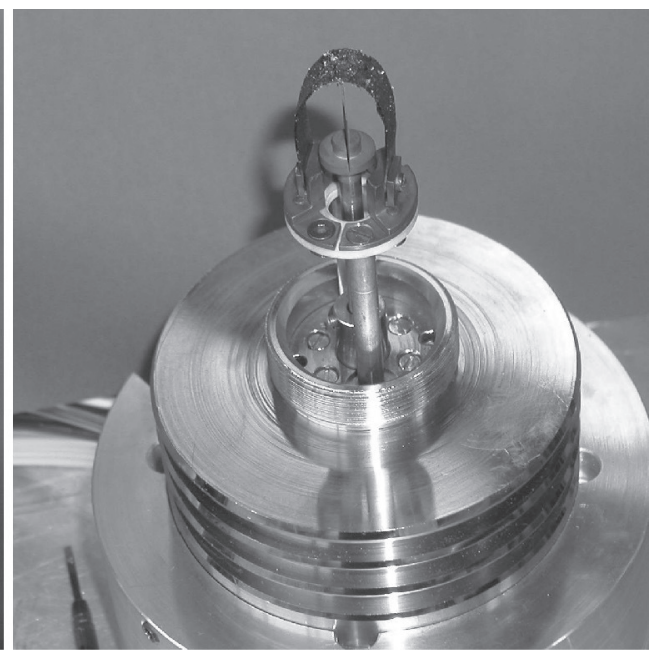

(b)

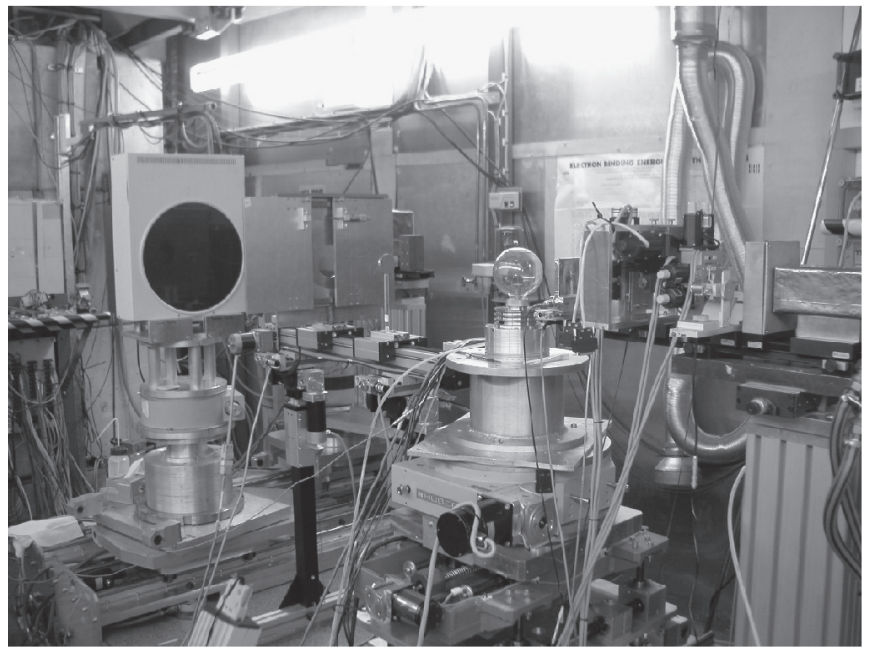

(c)

Fig. 18.6 Dome furnace mounted at the high energy beamline BW5, Hasylab at Desy, Hamburg.

The measurements were carried out with a primary slit system of $1 \times 1 \mathrm{~mm}^{2}$ and a wavelength of $0.124 \AA(100 \mathrm{keV})$.

The list of experiments include a texture measurement at room temperature, a measurement in the two phased region, texture measurements after cooling and phase analysis at different temperatures during heating and cooling. To verify the temperature a DTA analysis was included seeing the phase transition. The crosses show the measurement points of the in situ heating experiment with five points below the phase transition (bcc-phase), one point in the two phased region and two points in the fcc-phase region. 
Fig. 18.7 DTA of ST37 K including the measurement points of the in situ heating experiment.
Differential Thermal Analysis (DTA) of ST37K

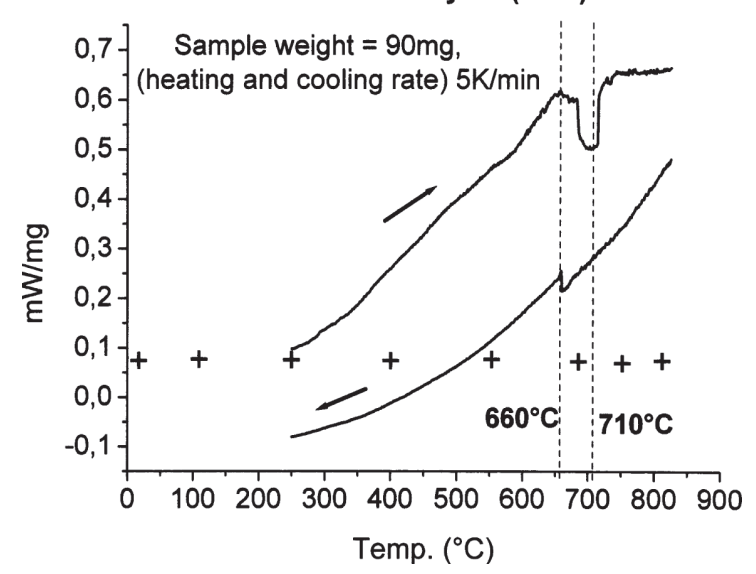

The phase analysis was carried out with single shots for each point with exposure times of 15 and $7 \mathrm{sec}$. Due to the readout time of about $90 \mathrm{sec}$., some additional measurements in the two-phased region with constant temperature and the cooling cylcle, where we also take data, the whole experiment takes about $50 \mathrm{~min}$. For quantitative texture analysis a set of individual measurements with $\Delta \omega$ of $5^{\circ}$ from $\omega=-90^{\circ}$ till $\omega=+90^{\circ}$ were done, which take much more time than the phase analysis. Therefor, the texture analysis was carried out at room temperature before and after heating and in the fcc-region.

\subsubsection{In situ High-Temperature Phase Analysis of ST37K}

In Fig. 18.8 one can see three typical image plate pictures for the different regions. Firstly, at room temperature the set of Debye-Scherrer rings (see Fig. 18.8 (a)) shows identical configuration as already explained for the powder spectra in Fig. 18.4 (a)., i.e. ferritic bcc. The main difference between the powder measurement and the solid material is that the intensity distribution along the DebyeScherrer ring varies. This is an indication of preferred orientation in the sample. Secondly, in Fig. 18.8 (c) the measurement at $820^{\circ} \mathrm{C}$ is shown. All Debye-Scherrer rings are spotty, which results from an unsufficient grain statistics. That means, at $820^{\circ} \mathrm{C}$ the material is much coarser. It can also bee seen that the positions of the Debye-Scherrer rings have changed. The diffraction pattern in Fig. 18.8 (c) represents now the finger print of the austenite phase with the hkl sequence (111), (200), (220), (311), (222).

In Fig. 18.8 (b) an overlap of both diffraction pattern were obtained. On one hand bcc ferrite is observed which has spotty Debye-Scherrer rings. That means less ferrite grains in the scattered volume. This can be explained by two reactions, one is the decreasing volume fraction by partly phase transition to the fcc-phase and second is a grain growth. A soft grain growth was observed during heating 
Fig. 18.8 Diffraction pattern of ST37 $\mathrm{K}$ at room temperature, at $680^{\circ} \mathrm{C}$ and at $820^{\circ} \mathrm{C}$.

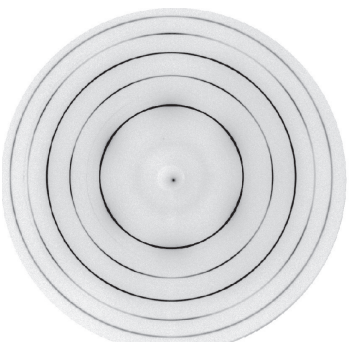

room temperature

(a)

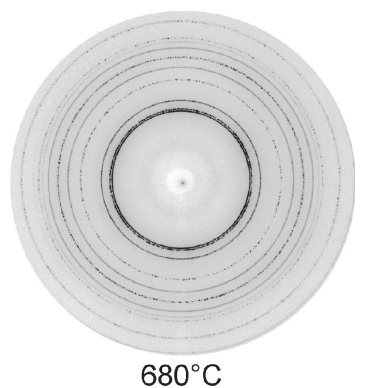

(b)

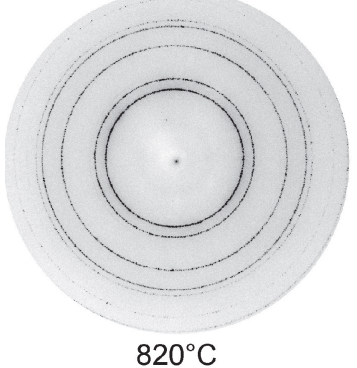

(c)

from room temperature to $550^{\circ} \mathrm{C}$. On the other hand one can see more or less good developed Debye-Scherrer rings of fcc austenite with sufficient grain statistics. Further heating to $750^{\circ} \mathrm{C}$ and $820^{\circ} \mathrm{C}$ result in grain growth of austenite grains that the Debye-Scherrer rings became spotty. It has to be noticed that the data analysis of two-phase steel is always influenced by the narrow Bragg-reflections of bcc (110) and fcc (111) as shown in Fig. 18.9. This picture is a zoom of a part of the complete Debye-Scherrer ring of Fig. 18.8 (b). In the case of the individual grains on the Debye-Scherrer ring the error bar of the lattice constant determination is much to high.

The data evaluation for lattice constants, thermal expansion coefficient and volume fraction in the two-phased region was carried out by MAUD [19] a free software package for Rietveld analysis [20] and quantitative texture determination. In a first step detector corrections for precize center of the mage plate picture and a misalignment by little tilt and rotation must be done. Due to the sample to detector distance of $100 \mathrm{~cm}$ small errors in the detector position can influence the lattice constants determination in the $\AA$-range. Next step is the calculate sum-diffraction

Fig. 18.9 Partly diffraction pattern of ST37 K at $680^{\circ} \mathrm{C}$ with narrow reflections of bcc $(110)$ and fcc (111).

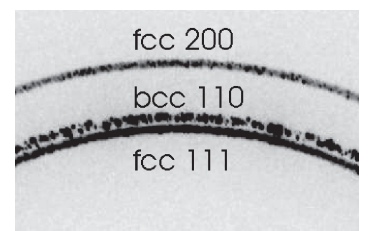


Fig. 18.10 Lattice constant of ferrite between room temperature and $550^{\circ} \mathrm{C}$.

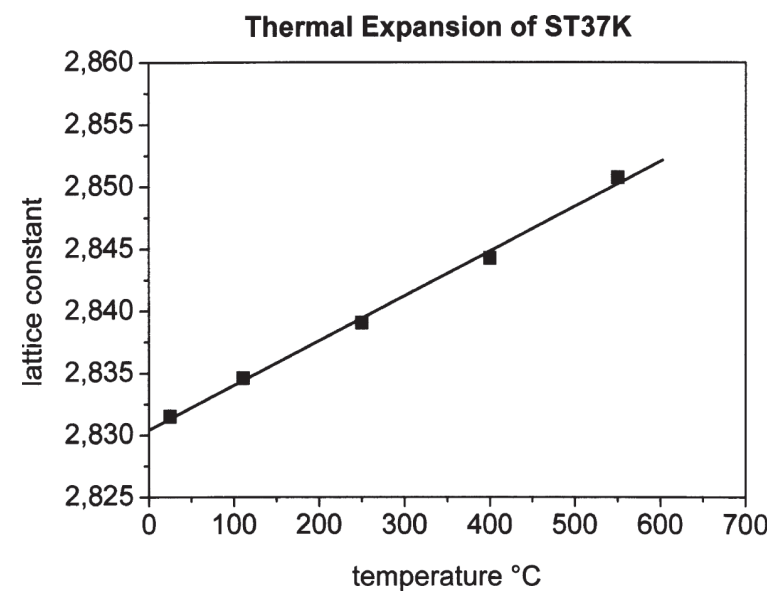

pattern to integrate over the whole Debye-Scherrer cone. A better grain statistics results and the influence of preferred orientation is minimized. A Rietveld refinement varried out with MAUD results in the lattice constants and the linear thermal expansion coeffizient. The linear thermal expansion coefficient $\alpha$ was calculated by the following equation to $0.9 \times 10^{-6} \mathrm{~K}^{-1}$.

$$
\alpha=1(\mathrm{~T})-1_{\mathrm{o}} / 1_{\mathrm{o}}-\mathrm{T}=\varepsilon_{\text {therm }} / \mathrm{T}
$$

Lattice parameters for ferrite vary from $2.83135 \AA$ at RT to $2.85076 \AA$ at $550^{\circ} \mathrm{C}$, see Fig. 18.10 .

The composition of the sample at $680^{\circ} \mathrm{C}$ was investgate for phase analysis. A first shot was made directly after reaching the tempearture of $680^{\circ} \mathrm{C}$ and a second measurement was carried out 5 min later. It results a ratio of $64.2 \%: 35.8 \%$ of ferrite : austenite for the first measurement, while after $5 \mathrm{~min}$ the austenite concentration increases to a ratio of $57.5 \%: 42.5 \%$ (ferrite : austenite).

\subsubsection{In situ High-Temperature Texture Analysis of ST37K}

As already pointed out, texture analysis is much more time consuming than phase analysis. Nevertheless synchrotron measurements are comparably fast due to the reduced number of single measurement, see Fig. 18.5. In the case of triclinic sample symmetry one needs a scan with 37 measurements having a resolution in $\omega$ of $5^{\circ}$. Two methods can be used to extract the pole figure data from the area detector data. In both cases one has to define a set of sections as shown in Fig. 18.11. $\gamma$ is the angle along the Debye-Scherrer ring and with $\Delta \gamma$ the resolution in $\gamma$ and the size of the section is defined. For our investigation we worked with $\Delta \gamma=5^{\circ}$ and $\Delta \gamma=1^{\circ}$. Sectioning can be done for each Debye-Scherrer ring (pole figure) separatly as realized by program systems of Sangbong Yi [21] or Ulf Garbe [22]. Each section is given by $2 \theta, \omega$ and $\gamma$ and the averaged reflection intensity of the 
Fig. 18.11 Sectioning of an area detector measurement for quantitative texture analysis $\left(\Delta \gamma=5^{\circ}\right)$.

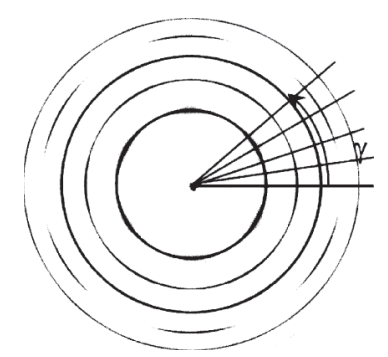

reflection hkl. A second possibility is to extract complete diffraction patterns for each section and to use a Rietveld refinement program like MAUD to obtain simultaneously all pole figures, which are included in the area detector data. The main difference is that Rietveld refinement needs the crystallographic data of the investigated material, while other programs work only with indexing the pole figures (hkl). In both cases a transformation has to be carried out to calculate the pole figure angles $(\alpha, \beta)$ from the reflection angle $2 \theta$, the sample rotation angle $\omega$ and the section angle $\gamma$. A detailed description of the transformation was give by Bunge and Klein [23].

Three texture measurements were carried out, at room temperature, at $820^{\circ} \mathrm{C}$ and at room temperature after cooling. A scanning routine with a set of 37 image plate pictures take between $60 \mathrm{~min}$.

Fig. 18.12 Ferrite pole figures measured at room temperature (counter levels $1.0,1.1,1.2,1.3 \ldots)$.

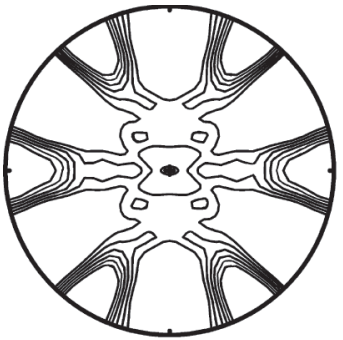

Ferrite (111)

$P \max =1.9 \mathrm{mrd}$

(a)

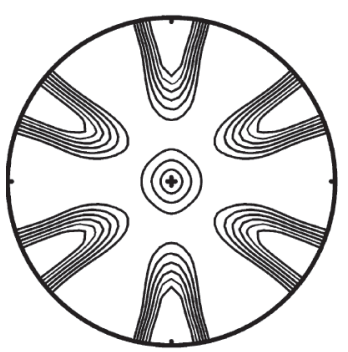

Ferrite (220)

$P \max =2.1 \mathrm{mrd}$

(c)

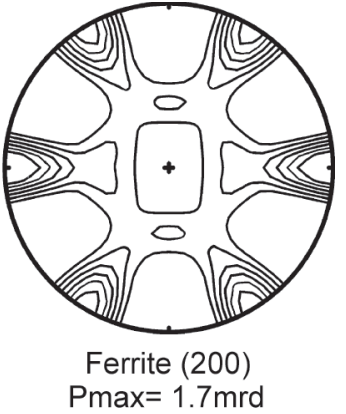

(b) 
Fig. 18.13 Austenite pole figures measured at $820^{\circ} \mathrm{C}$ (counter levels $1.0,1.1,1.2,1.3 \ldots)$.

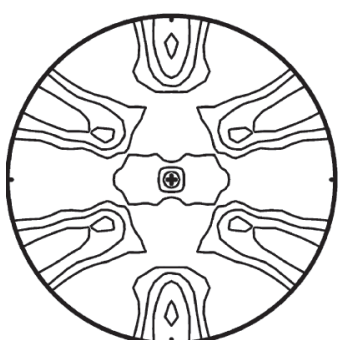

Austenite (111)

Pmax $=1.3 \mathrm{mrd}$

(a)

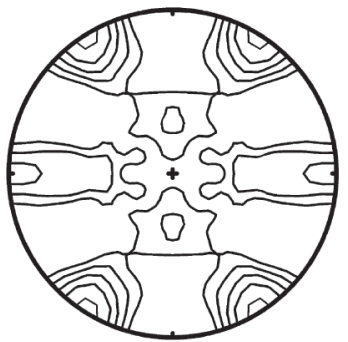

Austenite (220)

Pmax $=1.4 \mathrm{mrd}$

(c)

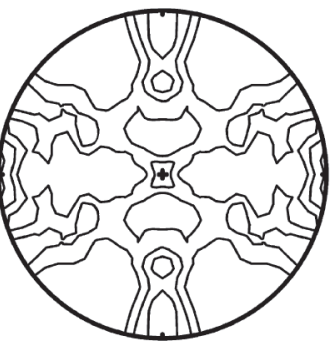

Austenite (200)

$P \max =1.3 \mathrm{mrd}$

(b)

The data transformation gave an equal angular set of values in $\alpha$ and $\beta$, such as a $5 \times 5$ matrix or a $5 \times 1$ matrix. Thereafter, the orientation distribution was calculated using the iterative series expansion method with a $\operatorname{lmax}=22$ as degree of series expansion. In Fig. 18.12 recalulated pole figure of ferrite measured at room temperature were shown. Mrd stands for multiple random. In the present case a weak texture was determined.

\subsection{Conclusion}

High-energy synchrotron radiation has many advantages over other technqiues such as conventional X-rays $(\mathrm{CuK} \alpha)$ or neutron diffraction. For crystallograhic texture analyses a broad spectrum of applications is available to measure small samples and local textures on one side and comparbly large semi-finished products on the other side. One point of interest is the combination of a high photon flux, an excellent brilliance and a low wavelength which is favoured for in situ experiments. Using an ST37 K sample in situ phase development, thermal expansion coefficient and texture transition has been demonstrated. The thermal expansion coefficient was caclulated to $\alpha=0.9 \times 10^{-6} \mathrm{~K}^{-1}$. Integration over complete DebyeScherrer ring allows the determination of volume fractions ferrite/austenite ratio in 
about 60 sec. counting time with minimized texture influence. With a MAR345 and a dome furnace one needs about $60 \mathrm{~min}$. to obtain quantitave textur data. First test with a new detector system and less readout time have shown that 5 to $10 \mathrm{~min}$ is realistic for a complete texture measurement. In future, a more detailed in situ textur analysis is possible than demonstrated in this paper. For the present example we detect an $\alpha \Leftrightarrow \gamma$ transformation following the Kurdjumov-Sachs model.

Acknowledgements This work has been funded by the German Ministry of Education and Research (BMBF) under the contract number $05 \mathrm{KS} 1 \mathrm{MCA} / 2$.

\section{References}

[1] O.V. Mishin, E.M. Lauridsen, N.C. Krieger Lassen, G. Brückner, T. Tschentcher, B. Bay, D. Juul Jensen and H.F. Poulsen, J. Appl. Crys. 33, 2000, 364.

[2] H.-R. Wenk and S. Grigull, J. Appl. Cryst. 36, 2003, 1040.

[3] H.J. Bunge, Adv. X-ray Analysis 47, 2004, 359.

[4] H.-G. Brokmeier and Sang Bong Yi, In: W. Reimers, A. Pyzalla, A. Schreyer, H. Clemens (eds.), Neutrons and Synchrotron Radiation in Engineering Materials Science, Wiley VCH Verlag, Weinheim, 2008 in press.

[5] H.-G. Brokmeier, Physica B: Condensed Matter 385-386, Part 1, 2006, 623.

[6] H.-G. Brokmeier: In: Advanced Materials 2005 eds: M. Farooque, S.A. Rizvi, J.A. Mirza KRL Rawalpindi Pakistan, 2007, 292

[7] H.-G. Brokmeier, S.B. Yi, B. Schwebke and J. Homeyer, Z. Kristallographie, Supl. 26, 2007.

[8] D. Juul Jensen, E.M. Lauridsen, L- Marulies, H.F. Poulsen, S. Schmidt, H.O. Sørensen and G.B.M. Vaughan, Materials Today 9, 2006, 18.

[9] H.-G. Brokmeier, B.Weiss, S.B. Yi, W. Ye, K.D. Liss and T. Lippmann, Mater. Sci. Forum 495-497, 2005, 131.

[10] B.D. Cullity, Elements of X-ray diffraction, Addison Wesley, 1978.

[11] H.- F. Kocks, C. Tome, H. R. Wenk, Texture and Anisotropy, Cambridge University Press 1998.

[12] J.F.H. Clusters: Philips Techn. Rundschau Vol. 7 (1942)

[13] G. Wassermann and J. Grewen: Texturen Metallischer Werkstoffe, Springer Verlag Berlin 1962.

[14] H. R. Wenk; Schweiz. Min. Petr. Mitt. 46, 1966, 518.

[15] K. Moras, A.H. Fischer, H. Klein and H.J. Bunge, J. Appl. Cryst. 33, 2000, 1162.

[16] S.-B. Yi, C.H.J. Davis, H.-G. Brokmeier, R.E. Bolmaro, K.U. Kainer and J. Homeyer, Acta Mat. 54, 549-562, 2006

[17] C.H.J. Davies, S.B. Yi , J. Bohlen, K.U. Kainer, H.-G. Brokmeier and J. Homeyer, Materials Science Forum 495-497, 2005, 1633.

[18] Sangbong Yi, H.-G. Brokmeier, J. Homeyer, Materials Science Forum 561-565, 2007, 183.

[19] L. Lutteroti, http://www.ing.unitn.it/ maud/

[20] H.M. Rietveld, J. Appl. Cryst. 2, 1969, 65.

[21] Sangbong Yi, PhD Theses TU Clausthal 2005

[22] Ulf Garbe private communication

[23] H.J. Bunge and H. Klein, Z. Metallkunde 87, 1996, 465.

[24] Z. Nishiyama, Martensitic Transformation, Academic Press, New York, 1978 


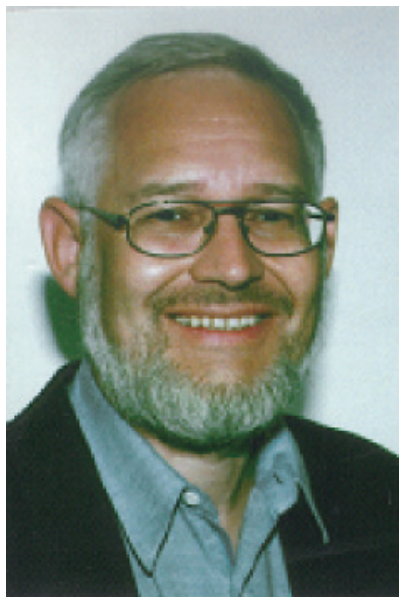

Heinz-Guenter Brokmeier Professor

Institute of Materials Science and Engineering Technical University Clausthal

Clausthal Agricolastr. 6

38678 Clausthal-Zellerfeld

Germany 